\title{
Collisional Ionization in Lithium Vapor Excited by Nanosecond Laser Pulses
}

\author{
M.A. Mahmoud*, A. El Tabal and M. Nady \\ Physics Department, Faculty of Science, Sohag University, 82524-Sohag, Egypt
}

(Received August 24, 2011; in final form April 4, 2012)

\begin{abstract}
We report a theoretical study of the collisional ionization processes which occur under $2 s \rightarrow 2 p$ excitation of lithium vapor excited with a nanosecond pulsed laser. The time evolution of the electron energy distribution function and the atomic ion density $\left(\mathrm{Li}^{+}\right)$as well as the molecular ion density $\left(\mathrm{Li}_{2}^{+}\right)$at different lithium vapor densities was investigated. The results show a nonlinear behavior of the energy spectra of the electrons created during the interaction of the laser with the lithium vapor. The nonlinear behavior results from superelastic collisions between the free electrons produced by collisional ionization and photoionization with $\operatorname{Li}(2 p)$. The results also show that a competition exists between the collisional ionization and photoionization processes in producing the atomic ion and the molecular ions.
\end{abstract}

PACS: $31.15 .-\mathrm{p}, 32.80 .-\mathrm{t}, 52.20 . \mathrm{Hv}$

\section{Introduction}

Collisional ionization and collisional excitation in thermal collisions between excited atoms and molecules in gases has been the subject of numerous studies during the last decade. The type of studies of elementary processes undertaken in low-temperature plasma physics is largely dictated by the needs of applied physics. This in turn presupposes rapid theoretical interpretation and purposeful utilization of the experimental results. In low-temperature plasma, collisional ionization is traditionally regarded as providing an effective channel for the production of atomic and molecular ions. Studies of such interactions are of great interest in a variety of fields, both fundamental and applied. In the plasma field, the most well known examples, include laser fusion, X-ray laser development, laser heating of magnetically confined plasmas [1].

Since the pioneering experiment on resonant laser plasma production in dense sodium vapor by Lucatorto and McIlrath [2], numerous ion investigations in $\mathrm{Li}$ [3-7], $\mathrm{Na}[8,9], \mathrm{Rb}[10,11]$, and $\mathrm{Cs}[12,13]$ vapors excited by $\mathrm{cw}$ or pulsed laser light have been reported previously. These studies provide insight into (i) the ionization mechanism, (ii) energy transfer mechanisms which are implied in or serve the ionization. Plasma formation in these experiments has been explained in terms of a propagating loop of superelastic collisions between neutral atoms and seed electrons $[14,15]$. The electrons gain kinetic energy

* corresponding author; e-mail: hameideg@yahoo.com in collisions with excited atoms and start runaway collisional ionization process. Recently, laser induced ionization has been shown to occur in vapors irradiated by nanosecond laser pulses $[16,17]$ when the ionization is only partial and does not exceed a few percent.

Due to the interest in laser excitation and ionization of lithium vapor, several experiments have been performed which study the microscopic processes that occur in laser-irradiated lithium vapor [3-6]. These microscopic processes dictate the collective behavior of the plasma or discharge and are therefore important for a complete understanding of the phenomena. Among the interactions that contribute to this complex environment are those involving direct photons, such as $h \nu+\mathrm{Li}$ and $h \nu+\mathrm{Li}_{2}$ and indirectly, for example $\operatorname{Li}(2 p)+\operatorname{Li}(2 p)$ energy pooling collisions.

On the other hand, resonance ionization spectroscopy of lithium vapor by laser radiation tuned to the first resonance lithium lines has been widely investigated in recent years [18-20] and this method is considered to be a useful technique for isotope separation because of its high selectivity [18].

Our interest in this work is primarily to study conditions (namely atom densities, irradiation time) for the collisional ionization and photoionization processes to help better understand ionization mechanisms in lithium vapor excited by a nanosecond pulsed laser. One of the principal assumptions made in the previous models [14-17] was that of a Maxwellian electron energy distribution function (EEDF). In the present work the rate coefficient of ionization and excitation is calculated with a non-equilibrium EEDF. 


\section{Theoretical model}

One assumption of the model is that the energy, $E_{\mathrm{c} 1}$, necessary to ionize the working atom is greater than twice the energy $E_{21}$ between the ground state and the first excited state, and less than the three times that energy, i.e. $2 E_{21}<E_{\mathrm{c} 1} \leq 3 E_{21}$. The alkali metals exhibit this property, and we will restrict our discussion to lithium. If the laser radiation is assumed to be suddenly applied at $t=0$, with a photon energy $h \nu=E_{21}$, then the subsequent chain of events can be viewed in the following section. Figure 1 shows the energy level diagram of states lithium atom involved in the present model.
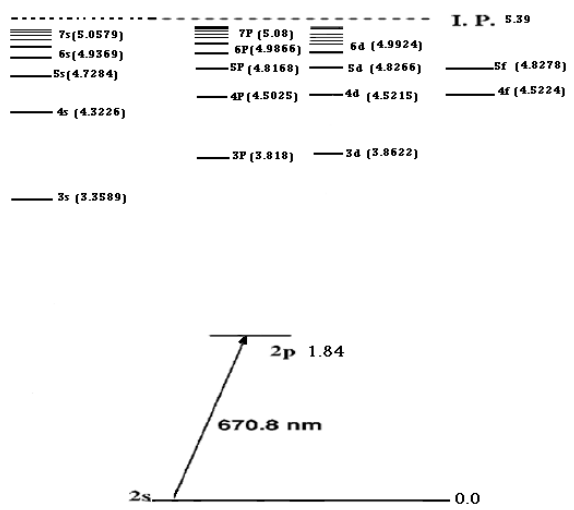

Fig. 1. Levels of lithium atom involved in the collisional processes which take place in the lithium vapor excited by laser radiation.

\subsection{Process considered}

a) The absorption of resonant photon

$$
\operatorname{Li}(2 s)+h \nu_{670.8} \rightarrow \operatorname{Li}(2 p) .
$$

b) Two-photon ionization of the $2 p$ level

$$
\mathrm{Li}(2 p)+2 h \nu_{670.8} \rightarrow \mathrm{Li}^{+}+\mathrm{e}^{-} .
$$

c) The associative ionization induced by laser of $2 p-2 p$ collision

$$
\mathrm{Li}(2 p)+\mathrm{Li}(2 p)+h \nu \rightarrow \mathrm{Li}_{2}^{+}\left({ }^{2} \Sigma_{2}(v, J)\right)+\mathrm{e}(\varepsilon) .
$$

d) The Penning ionization

$$
\mathrm{Li}(2 p)+\mathrm{Li}(n l) \rightarrow \mathrm{Li}^{+}+\mathrm{Li}(2 s)+\mathrm{e}\left(E_{2 p}-E_{n l}\right),
$$

where $E_{n l}$ is the binding energy of the $n l$ electron.

e) Laser-induced Penning ionization

$$
\mathrm{Li}(2 p)+\mathrm{Li}(2 p)+h \nu_{670.8} \rightarrow \operatorname{Li}(2 s)+\mathrm{Li}^{+}+\mathrm{e}^{-} .
$$

f) The energy pooling to the $3 d$ or $3 p$ level followed by photoionization.

$$
\begin{aligned}
& \operatorname{Li}(2 p)+\operatorname{Li}(2 p) \rightarrow \operatorname{Li}(3 d, 3 p)+\operatorname{Li}(2 s), \\
& \operatorname{Li}(3 d, 3 p)+h \nu_{670.8} \rightarrow \mathrm{Li}^{+}+\mathrm{e}^{-} .
\end{aligned}
$$

g) Superelastic collision (SEC). The superelastic collision is described by the collision of a low energy electron with an excited atom. This process can be written as

$$
\mathrm{Li}(n l)+\mathrm{e}\left(\varepsilon_{0}\right) \rightarrow \mathrm{Li}(2 s)+\mathrm{e}(\varepsilon) \text { where } \varepsilon>\varepsilon_{0} .
$$

h) Electron impact excitation. This process is the inverse of superelastic collision process

$$
\mathrm{Li}(n l)+\mathrm{e}(\varepsilon) \rightarrow \operatorname{Li}\left(n^{\prime} l^{\prime}\right)+\mathrm{e}\left(\varepsilon_{0}\right) \text { where } \varepsilon>\varepsilon_{0} .
$$

i) Electron impact ionization. When an electron gains enough energy (through superelastic collisions), which is equal to the ionization potential of the neutral atom, it can undergo an ionizing collision by electron impact process, resulting in a low energy electron of $\left(\varepsilon=\varepsilon_{1}+\varepsilon_{2}+\right.$ $\left.E_{\text {ion }}\right)$ and atomic ion

$$
\mathrm{Li}(n l)+\mathrm{e}(\varepsilon) \rightarrow \mathrm{Li}^{+}+\mathrm{e}\left(\varepsilon_{1}\right)+\mathrm{e}\left(\varepsilon_{2}\right)
$$

where $\varepsilon=\varepsilon_{1}+\varepsilon_{2}+E_{\text {ion }}$ and $E_{\text {ion }}$ is the ionization energy. j) Radiative recombination

$$
\mathrm{Li}^{+}+\mathrm{e}(\varepsilon) \rightarrow \mathrm{Li}(n l)+h \nu .
$$

k) Three-body recombination

$$
\mathrm{Li}^{+}+\mathrm{e}\left(\varepsilon_{1}\right)+\mathrm{e}\left(\varepsilon_{2}\right) \rightarrow \mathrm{Li}(n l)+\mathrm{e}(\varepsilon)
$$

where $3 s \leq n l \leq 7 d$.

For simplicity we have assumed that $\varepsilon_{1}=\varepsilon_{2}$ in the electron impact ionization and its inverse three-body recombination processes.

\subsection{Methods of calculations}

The rate of change of population density of states $2 s$, $2 p$ and the highly excited states $n l$ is given by

$$
\begin{aligned}
& \frac{\mathrm{d} N(2 s)}{\mathrm{d} t}=N(2 p)\left(R_{21}+A_{21}\right)-N(2 s) R_{12} \\
& \quad+N(2 p) \int n_{e}(\varepsilon) k_{21}(\varepsilon) \mathrm{d} \varepsilon-N(2 s) \int n_{e}(\varepsilon) k_{12}(\varepsilon) \mathrm{d} \varepsilon \\
& \quad+N(2 p) N(n) k_{P I}+\frac{1}{2} N^{2}(2 p) k_{E P} \\
& \quad-N(2 s) \int n_{e}(\varepsilon) k_{1 c}(\varepsilon) \mathrm{d} \varepsilon, \\
& \frac{\mathrm{d} N(2 p)}{\mathrm{d} t}=N(2 s) R_{12}-N(2 p)\left(R_{21}+A_{21}\right) \\
& \quad-N(2 p) \int n_{e}(\varepsilon) k_{21}(\varepsilon) \mathrm{d} \varepsilon+N(2 s) \int n_{e}(\varepsilon) k_{12}(\varepsilon) \mathrm{d} \varepsilon \\
& \quad-\frac{1}{2} N^{2}(2 p) k_{A I}-N(2 p) N(n) k_{P I}-\frac{1}{2} N^{2}(2 p) k_{E P} \\
& -N(2 p) \sigma_{2 c}(2) F^{2}-N(2 s) \int n_{e}(\varepsilon) k_{2 c}(\varepsilon) \mathrm{d} \varepsilon, \\
& \frac{\mathrm{d} N(n)}{\mathrm{d} t}=\sum_{n>m} n_{e}(\varepsilon) N(n) k_{n m}-\sum_{m>n} n_{e}(\varepsilon) N(n) k_{m n}(\varepsilon) \\
& \quad-\sum_{n>m} A_{n m} N(n)-\sum_{n} n_{e}(\varepsilon) N(n) k_{n c}(\varepsilon) \\
& -\sum_{n} N(2 p) N(n) k_{P I}+\frac{1}{2} N^{2}(2 p) k_{E P} \\
& -\sum_{n>2} N(n) \sigma_{n c}^{(1)} F
\end{aligned}
$$




$$
+N_{\mathrm{Li}}+n_{e}(\varepsilon) \sum_{n}\left[n_{e}(\varepsilon) k_{c n}(\varepsilon)+k_{R D}(\varepsilon)\right] .
$$

The rate of growth of the molecular ion and the atomic ion is given by

$$
\begin{aligned}
& \frac{\mathrm{d} N\left(\mathrm{Li}_{2}^{+}\right)}{\mathrm{d} t}=\frac{1}{2} N^{2}(2 p) k_{A I}, \\
& \frac{\mathrm{d} N\left(L i^{+}\right)}{\mathrm{d} t}=N(2 p) N(n) k_{P I}+N(2 p) \sigma_{2 c}(2) F^{2} \\
& \quad+\sum_{n>2} N(n) \sigma_{n c}^{(1)} F+\sum_{n} n_{e}(\varepsilon) N(n) k_{n c}(\varepsilon) .
\end{aligned}
$$

The electron energy distribution function equation is given by

$$
\begin{aligned}
& \frac{\mathrm{d} n_{e}(\varepsilon)}{\mathrm{d} t}=\sum_{m>n} n_{e}(\varepsilon) N(n) k_{n m}(\varepsilon) \\
& \quad-\sum_{m<n} n_{e}(\varepsilon) N(n) k_{m n}(\varepsilon)+\sum_{n} n_{e}(\varepsilon) N(n) k_{n c}(\varepsilon) \\
& \quad+\sum_{n} N(2 p) N(n) k_{P I}+N(2 p) \sigma_{2 c^{(2)}} F^{2} \\
& \quad+\sum_{n>2} N(n) \sigma_{n c}^{(1)} F+\frac{1}{2} N^{2}(2 p) k_{A I} \\
& \quad-N_{\mathrm{Li}^{+}} n_{e}(\varepsilon) \sum_{n}\left[n_{e}(\varepsilon) k_{c n}(\varepsilon)+k_{R D}(\varepsilon)\right] .
\end{aligned}
$$

The normalization conditions are

$$
\begin{gathered}
N_{0}=\sum_{n} N(n)+N\left(\mathrm{Li}^{+}\right), \\
\int_{0}^{\infty} n_{e}(\varepsilon) \varepsilon^{1 / 2} \mathrm{~d} \varepsilon=1,
\end{gathered}
$$

where $N_{0}$ is the density of Li atomic vapor. $N(2 s), N(2 p)$ and $N(n)$ are the population density of levels $2 s, 2 p$ and $n l$, respectively, $n_{e}(\varepsilon)$ represents the free electron density as a function of electron energy $\varepsilon, R_{12}$ is the stimulated absorption rate coefficient from $2 s$ to $2 p$ level $\left(\mathrm{s}^{-1}\right), R_{21}$ is the stimulated emission rate coefficient from $2 p$ to $2 s$ level $\left(\mathrm{s}^{-1}\right), A_{n m}$ is the Einstein coefficient for spontaneous emission for the transition $n \rightarrow m\left(\mathrm{~s}^{-1}\right)$, $k_{E P}$ represents the energy pooling collisions rate coefficient $\left(\mathrm{cm}^{3} \mathrm{~s}^{-1}\right), k_{A I}$ represents the association ionization rate coefficient $\left(\mathrm{cm}^{3} \mathrm{~s}^{-1}\right) k_{P I}$ represents penning ionization rate coefficient for level $n\left(\mathrm{~cm}^{3} \mathrm{~s}^{-1}\right), k_{21}$ represents the electron collision rate coefficient for transition from $(2 p \rightarrow 2 s)$ as a function of electron energy $(\varepsilon)\left(\mathrm{cm}^{3} \mathrm{~s}^{-1}\right)$, $k_{12}$ represents the electron collision rate coefficient for transition from $(2 \rightarrow 2 p)$ as a function of electron en$\operatorname{ergy}(\varepsilon)\left(\mathrm{cm}^{3} \mathrm{~s}^{-1}\right), k_{2 c}$ represents electron collision ionization rate coefficient for level $2 p\left(\mathrm{~cm}^{3} \mathrm{~s}^{-1}\right), k_{1 c}$ represents electron collision ionization rate coefficient for level $2 s\left(\mathrm{~cm}^{-1} \mathrm{~s}^{-1}\right), k_{n c}(\varepsilon)$ represents electron collision ionization rate coefficient for level $n\left(\mathrm{~cm}^{3} \mathrm{~s}^{-1}\right), k_{n m}$ represents the electron collision rate coefficient for transition from $(n \rightarrow m)$ as a function of electron energy $(\varepsilon)\left(\mathrm{cm}^{3} \mathrm{~s}^{-1}\right)$, $k_{m n}(\varepsilon)$ represents the electron collision rate coefficient for transition from $(m \rightarrow n)$ as a function of electron energy $\varepsilon\left(\mathrm{cm}^{3} \mathrm{~s}^{-1}\right), \sigma_{n c}^{(1)}$ represents the single photon ionization cross-section, $\sigma_{2 c}^{(2)}$ represents the two-photon ionization cross-section, $F$ is the photon flux (photon $\mathrm{s}^{-1} \mathrm{~cm}^{-2}$ ), $k_{c n}$ represents the three body recombination rate coefficient for $n$ level $\left(\mathrm{cm}^{6} \mathrm{~s}^{-1}\right), k_{R D}$ represents the radiative

\begin{tabular}{|c|c|c|}
\hline Process & Cross section & Reference \\
\hline $\operatorname{Li}(2 p)+2 h \nu \rightarrow \mathrm{Li}^{+}$ & $20 \times 10^{-18} \mathrm{~cm}^{2}$ & {$[22]$} \\
\hline $\mathrm{Li}(2 p)+\mathrm{Li}(2 p)+h \nu \rightarrow \mathrm{Li}_{2}^{+}+\mathrm{e}^{-}$ & $5 \times 10^{-20} \mathrm{~cm}^{2}$ & {$[23]$} \\
\hline $\operatorname{Li}(2 p)+\operatorname{Li}(2 p) \rightarrow \operatorname{Li}(3 p, 3 d)+\operatorname{Li}(2 s)$ & $7.8 \times 10^{-15} \mathrm{~cm}^{2}$ & $(12]$ \\
\hline $\mathrm{Li}(2 p)+\mathrm{Li}(2 p)+h \nu_{670.8} \rightarrow \operatorname{Li}(2 s)+\mathrm{Li}^{+}+\mathrm{e}^{-}$ & $91 \times 10^{-44} \mathrm{~cm}^{4} \mathrm{~s}$ & [24] \\
\hline \multicolumn{3}{|c|}{ Cross sections of photoionization for lithium $(\mathrm{Mb})$} \\
\hline $3 s$ & 1.48 & {$[22]$} \\
\hline $4 s$ & 1.40 & {$[22]$} \\
\hline $5 s$ & 1.33 & [22] \\
\hline $2 p$ & 15.20 & {$[22]$} \\
\hline $3 p$ & 28.10 & [22] \\
\hline $4 p$ & 41.7 & [22] \\
\hline $5 p$ & 56.2 & [22] \\
\hline $3 d$ & 18.2 & [22] \\
\hline $4 d$ & 36.2 & [22] \\
\hline
\end{tabular}
recombination rate coefficient $\left(\mathrm{cm}^{3} \mathrm{~s}^{-1}\right), N_{\mathrm{Li}^{+}}$represents the density of atomic ions, $N_{\mathrm{Li}_{2}^{+}}$represents the density of molecular ions. Let us note that the factor $1 / 2$ with $k_{E P}$ and $k_{A I}$ corrects for possible double counting of each colliding pair of identical particles [21].

The rate coefficients of collisional ionization processes and the cross-sections of the photoionization processes in our model is indicated in Table. 


\subsection{Lithium data}

The computational model consists of a large number of rate equations describing the densities of the various excited states of the lithium atom, the atomic and molecular ions and the energy distribution of the free electrons which are created during the interaction of lithium vapor with nanosecond pulsed laser. The rate equation for the electron energy distribution (8) is divided into 54 equations, where the ionization energy of $\mathrm{Li}=5.39 \mathrm{eV}$ and the energy step equal $0.1 \mathrm{eV}$.

To calculate the electron energy distribution function, numerous data have to be compiled from the literature to be used in the Boltzmann equation computational code $[25,26]$. By the use of suitable approximations for the energy dependence of the cross-sections, the rate coefficients can be expressed by simple analytical formula. The rate coefficients $k_{n c}, k_{c n}$ is given by Vriens and Smeets [27]. The rate coefficient $k_{n m}$ and $k_{m n}$ have been taken from Ref. [15]. For the radiative recombination rate coefficient we have used the empirical formula given by Drawin [28].

\section{Results and discussion}

The set of equations specified in Sect. 2.2 are solved numerically under the experimental conditions of Skenderovic et al. [5]. In this instance the lithium number density was varied in the range from $10^{14}$ to $10^{16} \mathrm{~cm}^{-3}$ and the vapor temperature was $650 \mathrm{~K}$. The pulse half-width of the laser beam is about $20 \mathrm{~ns}$ and the repetition rate can be varied from 1 to $10 \mathrm{~Hz}$. The typical energy of single pulse $(E)$ is $2 \mathrm{~mJ}$ and the laser beam diameter was about $2.5 \mathrm{~mm}$. The laser intensity $(I)$ is given by

$$
I=\frac{E}{\tau \times \pi r^{2}}
$$

where $\tau$ is the laser pulse duration and $r$ is the laser beam radius. Under these conditions, the laser light was tuned to the first resonance $2 s \rightarrow 2 p$, at $\lambda=670.8 \mathrm{~nm}$. We calculate (i) the time evolution of the electron energy distribution function for different values of lithium atom, (ii) the time evolution of the atomic ion for different values of lithium atomic vapor and (iii) the time evolution of the molecular ion for different values of lithium atomic vapor.

\subsection{The time evolution of electron energy distribution function}

The time evolution of the electron energy distribution function at lithium vapour density equal to $1 \times 10^{15} \mathrm{~cm}^{-3}$ is shown in Fig. 2. From this figure we notice that the spectral structure is observed with certain peaks $(A, B$, $C, D, E$, and $F$ ) lying at energies $0.2,1,1.5,2.25,2.75$, and $3.75 \mathrm{eV}$, respectively. The energy distribution of the electrons produced in peak $A$ is centered at a mean energy $E_{m} \approx 0.2 \mathrm{eV}$ by single photoionization of $3 p$ and $3 d$, laser induced penning ionization of $2 p$ and two-photon ionization of $2 p$ which are described by the following equations:

$$
\mathrm{Li}(2 p)+2 h \nu_{670.8} \rightarrow \mathrm{Li}^{+}+\mathrm{e}^{-}
$$

two-photon ionization),

$$
\mathrm{Li}(2 p)+\mathrm{Li}(2 p)+h \nu \rightarrow \mathrm{Li}^{+}+\mathrm{Li}(2 s)+\mathrm{e}^{-}
$$

laser-induced Penning ionization).

The peak $B$ corresponds to electrons produced by the laser induced associative ionization process

$$
\mathrm{Li}(2 p)+\mathrm{Li}(2 p)+h \nu \rightarrow \mathrm{Li}_{2}^{+}+\mathrm{e}^{-} .
$$

The peak $C$ corresponds to single-photon ionization

$$
\mathrm{Li}(3 p, 3 d)+h \nu_{670.8} \rightarrow \mathrm{Li}^{+}+\mathrm{e}^{-} .
$$

The peak $D$ corresponds to the electrons produced by the Penning ionization of $\operatorname{Li}(3 d)$ which is described by the following collision:

$$
\mathrm{Li}(2 p)+\mathrm{Li}(2 p) \rightarrow \mathrm{Li}^{+}+\mathrm{Li}(2 s)+\mathrm{e}^{-}\left(2 E_{2 p}-E_{\text {ion }}\right) .
$$

The two peaks $E, F$ are attributed to the superelastic collisions

$$
\mathrm{Li}(n l)+\mathrm{e}\left(\varepsilon_{0}\right) \rightarrow \operatorname{Li}(2 s)+\mathrm{e}(\varepsilon), \text { where } \varepsilon>\varepsilon_{0} .
$$

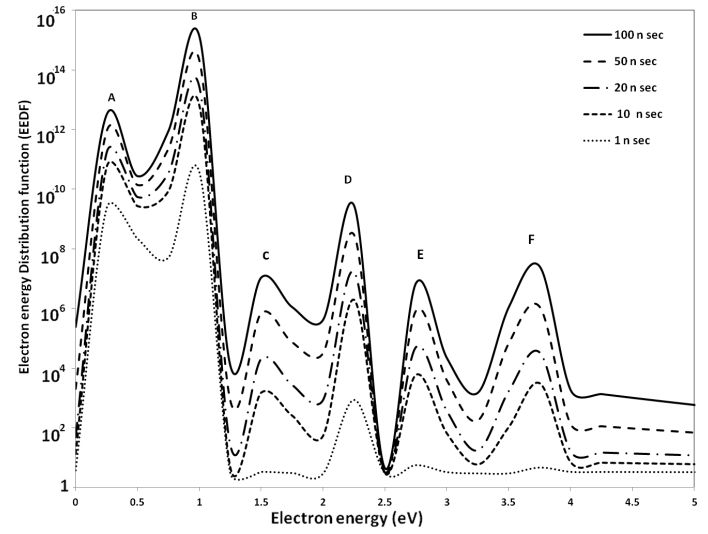

Fig. 2. Time development of the electron energy distribution function in lithium vapor with excitation by pulsed laser at vapor density equal to $1 \times 10^{15} \mathrm{~cm}^{-3}$.

The dependence of the electron energy distribution function on the lithium vapor density is indicated in Fig. 3. This figure enables us to study the evolution of the electrons seeding and growing processes. Also this figure confirmed the observed peaks ( $A, B, C, D, E$ and $F$ ), which change in height as the lithium vapor density increases. However the interesting point from our perspective is that the collisional ionization and photoionization are the dominant processes during the plasma formation in laser excited lithium vapor [3-6]. Also, Gorbunov et al. [6] have demonstrated that excitation processes from $\operatorname{Li}(2 p)$ can play a significant role in determining the EEDF and the major influence is the excitation of $3 \mathrm{~s}$ state having the threshold of $\Delta_{2 p-3 s}=1.5 \mathrm{eV}$.

\subsection{The time evolution of atomic ion $\mathrm{Li}^{+}$}

The growth rate of the $\mathrm{Li}^{+}$as a function of time for different values of lithium vapor density is given by Fig. 4. 


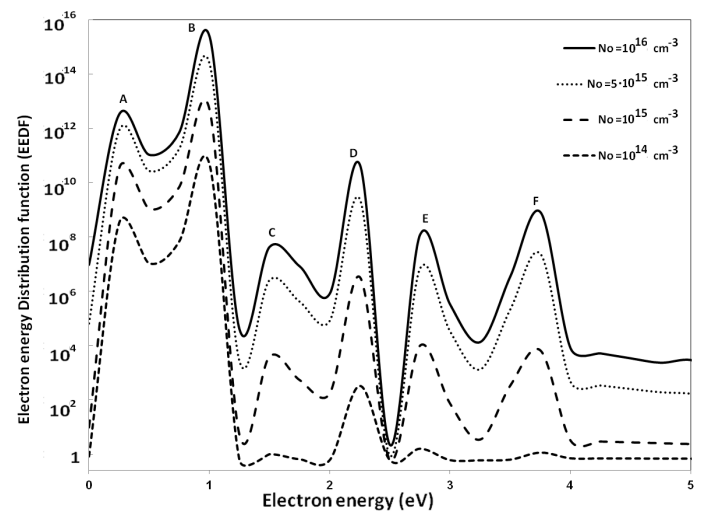

Fig. 3. The dependence of the electron energy distribution function on the lithium vapor density after $20 \mathrm{~ns}$ from the laser pulse.

From this figure it can be seen that the $\mathrm{Li}^{+}$shows a fast increase during the early stages of the interaction which increases up to $20 \mathrm{~ns}$. The curves show almost the same behavior with descending values of the lithium vapor density for different irradiation times. This behavior is due to the $\mathrm{Li}^{+}$mainly produced by the photoionization and laser induced Penning ionization processes [3-5].

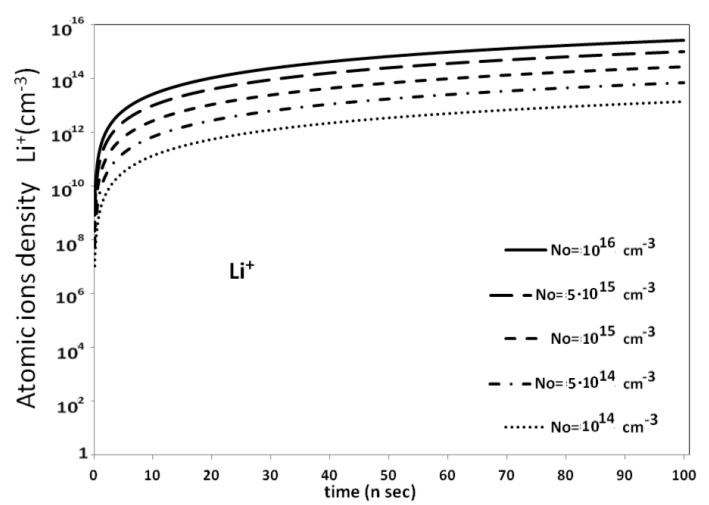

Fig. 4. Time evolutions of atomic ions $\left(\mathrm{Li}^{+}\right)$at different values of lithium vapor densities.

\subsection{The time evolution of molecular ion $L i_{2}^{+}$}

The growth rate of the molecular ions $\mathrm{Li}_{2}^{+}$as a function of time is illustrated in Fig. 5 for different values of lithium vapor density. From this figure we can see that the $\mathrm{Li}_{2}^{+}$density increases rapidly during the period $0.1 \mathrm{~ns}$ up to $2 \mathrm{~ns}$ followed by a slow increase up to $20 \mathrm{~ns}$ for the different values of the lithium vapor density. The explanation of the linear growth can be described as follows. During the early stages, the molecular ions are created at a high rate through associative ionization process. Immediately, after this time the $\mathrm{Li}_{2}^{+}$density shows a slow increase during the late stages of the irradiation time. An understanding of this behavior can be attained by considering that the main processes for producing these molecular ions are associative ionization [4].

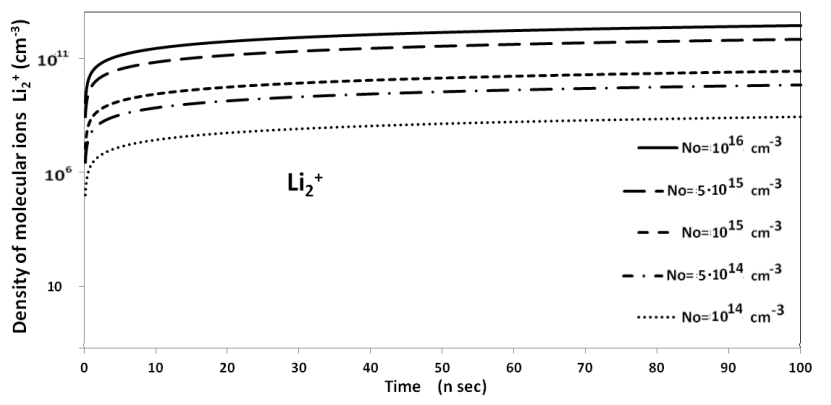

Fig. 5. Time evolution of molecular ions $\left(\mathrm{Li}_{2}^{+}\right)$at different values of lithium vapor densities.

\subsection{The time evolution of the total concentration of electrons}

The growth rate of electrons $N_{e}$ as a function of time is indicated in Fig. 6 for different values of lithium vapor density. From this figure we can see that the electron density increases up to $30 \mathrm{~ns}$. Immediately after this time the electron density shows a slow increase during the late stages of the interaction. The explanation of this growth can be described as follows. During the early stages of the interaction, a rapid growth of free electrons appears due to two-photon ionization of the resonance level and laser induced Penning ionization. These electrons now give rise to an exponential growth due to both direct collisional ionization of the resonance level and single photon ionization of the populated intermediate levels. Beyond this time, the growth rate of the electrons becomes slower due to the competition between the generation of the electrons through Penning ionization or photoionization, as well as collisional ionization of the ground state and excited losses of the ions by radiative recombination and three-body recombination [5].

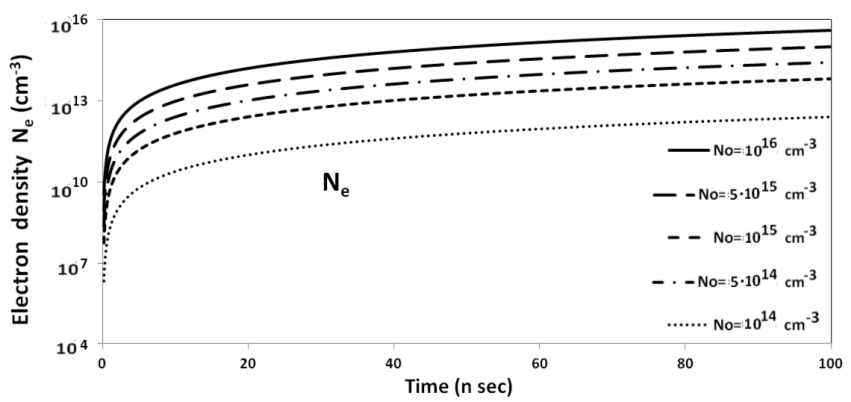

Fig. 6. Time evolution of electron density $\left(N_{e}\right)$ at different values of lithium vapor densities. 


\subsection{The $N_{e}, L i^{+}$and $L i_{2}^{+}$as a function of the lithium vapor density}

The growth rate of the $N_{e}, \mathrm{Li}^{+}$and $\mathrm{Li}_{2}^{+}$as a function of the lithium vapor density is shown in Fig. 7. The curves show almost the same behavior and the atomic ion $\mathrm{Li}^{+}$is greater than the molecular ion $\mathrm{Li}_{2}^{+}$. This behavior is due to the rate coefficient of photoionization and Penning ionization processes being greater than the rate coefficient of the associative ionization process. Skenderovic et al. [5] reported that, after the absorption of a resonant photon,

$$
\operatorname{Li}(2 s)+h \nu_{670.8} \rightarrow \operatorname{Li}(2 p),
$$

ions can be created by following paths. The first is a two photon ionization of the $2 p$ level

$$
\mathrm{Li}(2 p)+2 h \nu_{670.8} \rightarrow \mathrm{Li}^{+}+\mathrm{e}^{-} .
$$

The second path is energy pooling to the $3 d$ or $3 p$ level followed by photoionization:

$$
\begin{aligned}
& \operatorname{Li}(2 p)+\operatorname{Li}(2 p) \rightarrow \operatorname{Li}(3 d, 3 p)+\operatorname{Li}(2 s), \\
& \operatorname{Li}(3 d, 3 p)+h \nu_{670.8} \rightarrow \mathrm{Li}^{+}+\mathrm{e}^{-} .
\end{aligned}
$$

The third one is radiation-assisted Penning ionization

$$
\mathrm{Li}(2 p)+\mathrm{Li}(2 p)+h \nu_{670.8} \rightarrow \mathrm{Li}(2 s)+\mathrm{Li}^{+}+\mathrm{e}^{-} .
$$

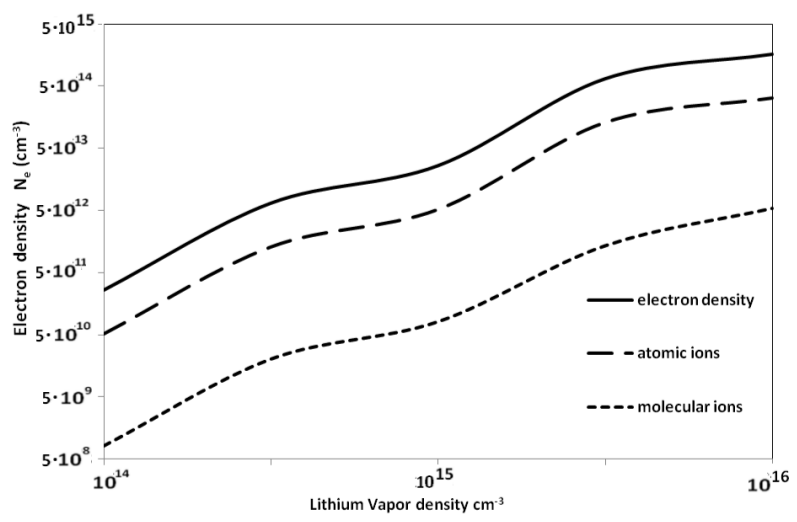

Fig. 7. Variation of electrons density $N_{e}$, atomic ions $\mathrm{Li}^{+}$and molecular ions $\mathrm{Li}_{2}^{+}$density with lithium vapor density $N_{0}$ at $20 \mathrm{~ns}$ from the laser pulse.

On the other hand, photoionization can play a remarkable role at a low ionization degree of the medium, when the basic mechanism of the $\operatorname{Li}(2 p, 3 d)$ population are energy-pooling [6, 7] collisions: $\operatorname{Li}(2 p)+\operatorname{Li}(2 p) \rightarrow$ $\mathrm{Li}(3 d, 3 p)+\mathrm{Li}(2 s)$. Through this process electron excitation prevails when $k_{E P} N(2 p)>k_{n m} n_{e}$. As far as we know, there is no definite value for $k_{E P}$ for lithium. The considered reaction is an endothermic one. For the energy pooling reaction with resonant $\mathrm{Cs}$ atoms with a similar energy gap $\left(\Delta E_{p} \approx 0.18 \mathrm{eV}\right)$ the rate coefficients are of an order $k_{E P} \approx 10^{-12} \mathrm{~cm}^{3} \mathrm{~s}^{-1}$ [12]. In fact, the two processes, (superelastic transfer to the free electrons and energy pooling collisions) work in tandem to create quasicontinuous population inversions. The indirect en- ergy transfer through electrons is indeed faster and more efficient compared with direct energy pooling. However, once a high population of states $3 p$ and $3 d$ is achieved, the radiative loss increases since these states have short radiative lifetimes [5-7].

\section{Conclusions}

We have carried out a computational study on the influence of collisional ionization and collisional excitation processes on the electron energy distribution function, as well as the molecular ions and atomic ions in laser-excited lithium vapor. In particular, the time evolution of the electron density, the atomic and the molecular ion at different values of lithium density was investigated. The results clarified that there is a rapid growth of free electrons due to two-photon ionization of resonance level and laser induced ionization. The electrons created by these initial processes rapidly gain energy through superelastic collisions with laser sustained resonance state population, effectively quenching that state, as well as the energy pooling collisions which play role in populating the $3 p$ and $3 d$ states. In general, it is shown that for a reasonable amount of laser energy tuned to the first resonance state of lithium a rapid ionization is possible through photoionization and collisional ionization processes and lithium plasmas of high density could be produced.

\section{References}

[1] A.N. Klyucharev, Phys. Usp. 36, 486 (1993).

[2] T.B. Lucatorto, T.J. McIlrath, Phys. Rev. Lett. 37, 428 (1976).

[3] D. Veza, C.J. Sansonetti, Z. Phys. D, At. Mol. Clust. 22, 463 (1992).

[4] I. Labazan, S. Milosevic, Eur. Phys. J. D 8, 41 (2000).

[5] H. Skenderovic, I. Labazan, S. Milosevic, G. Pichler, Phys. Rev. A 62, 052707 (2000).

[6] N.A. Gorbunov, A.S. Melnikov, I. Smurov, I. Bray, J. Phys. D, Appl. Phys. 34, 1379 (2001).

[7] Ch. He, R.A. Bernheim, Chem. Phys. Lett. 190, 494 (1992).

[8] V. Horvatic, Fizika A 12, 97 (2003).

[9] T. Stacewicz, Acta Phys. Pol. A 84, 259 (1993).

[10] A. Ekers, M. Glodz, J. Szonert, B. Bieniak, K. Fronc, T. Radeltiski, Eur. Phys. J. D 8, 49 (2000).

[11] R. Beuc, M. Movre, V. Horvatic, C. Vadla, O. Dulieu, M. Aymar, Phys. Rev. A 75, 032512 (2007).

[12] F. de Tomas, S. Milosevic, P. Verkerk, A. Fioretti, M. Allegrini, Z.J. Jabbour, J. Huennekens, J. Phys. B, At. Mol. Opt. Phys. 30, 4991 (1997).

[13] V. Horvatic, T. Correll, N. Omenetto, C. Vadla, J. Winefordner, Spectrochim. Acta Part B 61, 1260 (2006).

[14] R.M. Measures, J. Appl. Phys. 48, 2673 (1977).

[15] R.M. Measures, P.G. Cardinal, Phys. Rev. A 23, 804 (1981). 
[16] T. Stacewicz, G. Topulos, Phys. Scr. 38, 560 (1988).

[17] T. Stacewicz, W. Latek, Phys. Scr. 38, 658 (1990).

[18] T. Arisawa, Y. Maruyama, Y. Suzuki, K. Shiba, Appl. Phys. B 28, 73 (1982).

[19] I.E. Olivares, A.E. Durate, E.A. Saravia, F.J. Durate, Appl. Opt. 41, 2973 (2002).

[20] M. Saleem, S. Hussain, M. Rafiq, M.A. Baig, J. Appl. Phys. 100, 053111 (2006).

[21] N.N. Bezuglov, A.N. Klyucharev, V.A. Sheverev, J. Phys. B, At. Mol. Phys. 20, 2497 (1987).

[22] M. Aymar, E. Luc-Koenig, F. Combet Farnoux, J. Phys B, At. Mol. Phys. 9, 1279 (1976).
[23] A.V. Hellfeld, J. Caddick, J. Weiner, Phys. Rev. Lett. 40, 1369 (1978).

[24] S. Geltman, J. Phys. B 10, 3057 (1977).

[25] Y.E.E. Gamal, M.A. Mahmoud, H.A. Abd El Rahman, J. Quant. Spectrosc. Radiat. Transfer 90, 29 (2005).

[26] M.A. Mahmoud, J. Phys. B, At. Mol. Opt. Phys. 38, 1545 (2005).

[27] L. Vriens, A.H.M. Smeets, Phys. Rev. A 22, 940 (1980).

[28] W.H. Drawin, Raport Euratom CEAFC, 1967, p. 38. 